\title{
On Some Edge Rotation Distance Graphs
}

\author{
Medha Itagi Huilgol ${ }^{1} \&$ Chitra Ramaprakash ${ }^{2}$ \\ ${ }^{1}$ (Central College Campus, Bangalore University, India) \\ ${ }^{2}$ (Central College Campus, Bangalore University, India)
}

\begin{abstract}
The concept of edge rotations and distance between graphs was introduced by Gary Chartrand et.al [1].A graph $G$ can be transformed into a graph $H$ by an edge rotation if $G$ contains distinct vertices $u, v$ and $w$ such $u v \in E(G)$ and $u w \notin E(G)$ and $H \cong G-u v+u w$. In this case, $G$ is transformed into $H$ by" rotating" the edge uv of $G$ into $u w$. In this paper we consider rotations on generalized Petersen graphs and minimum selfcentered graphs. We have also developed algorithms to generate distance degree injective (DDI) graphs and almost distance degree injective (ADDI) graphs from cycles using the concept of rotations followed by some general results.
\end{abstract}

Keywords: ADDI graphs, average eccentricity, DDI graphs, edge rotations, edge rotation distance graphs, Generalized Petersen graph, r-distance graph.

\section{Introduction}

Unless mentioned otherwise, for terminology and notation the reader may refer to Buckley and Harary[23] and Chartrand and Zang [24], new ones will be introduced as and when found necessary.

In this paper, by a graph $G$, we mean a simple, undirected, connected graph without self- loops. The order and size are respectively the number of vertices denoted by $n$ and number of edges denoted by $m$.

The distance $d(u, v)$ between any two vertices $u$ and $v$, of $G$, is the length of the shortest path between $u$ and $v$. The eccentricity $e(u)$ of a vertex $u$ is the distance to a farthest vertex from $u$. The maximum and the minimum eccentricity amongst the vertices of $G$ are respectively called the diameter $\operatorname{diam}(G)$ and radius $\operatorname{rad}(G)$. If $\operatorname{diam}(G)=\operatorname{rad}(G)$, then the graph $G$ is said to be self-centered graph. If $d(u, v)=e(u),(v \neq u)$ then we say that $v$ is an eccentric vertex of $u$.

The distance degree sequence (dds) of a vertex in a graph $G=(V, E)$ is the list of number of vertices at distance $1,2, \ldots e(v)$ in that order, where $e(v)$ denotes the eccentricity of $v$. Thus the sequence $\left(d_{i_{0}}, d_{i_{1}}, d_{i_{2}}, \ldots, d_{i_{j}}\right)$, is the distance degree sequence $(d d s)$ of the vertex $v_{i}$ in $G$ where $d_{i_{j}}$ denotes the number of vertices at distance $j$ from $v_{i}$.

The concept of distance degree regular (DDR) graphs was introduced by Bloom et al. [26] as the graph for which all vertices have the same distance degree sequence. This was further studied by Bloom et al. [25], Halberstam et al. [27], Itagi Huilgol et al. [2], [3], [4].

The other extreme of distance degree regular $(D D R)$ graphs is the distance degree injective (DDI) graphs. The concept of distance degree injective graphs was introduced by Bloom et al. in [25]. A graph $G$ is said to be a distance degree injective (DDI) graph if no two of its vertices have the same distance degree sequence. In literature, characterizations of both $D D R$ and $D D I$ graphs are not known. But there are several particular cases, [26], [27], [2], [3], [4], [28], etc. So the construction of distance degree injective graphs or the almost distance degree injective graphs $(D D I / A D D I)$ is also a challenging one. In [3] Itagi Huilgol et al. have constructed higher order DDI graphs using products. Fast generation of cubic graphs was done by Brinkmann [5]. In [6] Itagi Huilgol et al. have introduced the concept of Almost Distance degree Injective (ADDI) graphs. A graph $\mathrm{G}$ of order $\mathrm{n}$ is said to be ADDI or almost DDI if $n-2$ vertices have different distance degree sequence and two vertices have the same distance degree sequence.

The concept of distance between isomorphism classes of graphs was introduced by Zelinka [7] which was later extended to trees [8] also. Based on these two papers the concept of rotations called 'edge rotations' and the distance between such graphs was introduced by Chartrand et al. [1]. A graph $G$ can be transformed into a graph $H$ be an edge rotation given by $H \cong G-u v+u w$ where $u, v$ and $w$ are distinct vertices of G such that $u v \in E(G)$ and $u w \notin E(G)$. Later in [9] Zelinka gave a comparison of various distance for the isomorphism classes of graphs and trees, which was based on the concept of edge rotations. It was also showed that this distance is a metric by Balaz et al. in [10].

Zelinka studied various aspects using the concept of distance between graphs and edge rotations in [11], [12] and [13]. The concept of "edge move" was defined by Balaz et al. [10] and is defined in this manner. A graph $G$ can be transformed into a graph $H$ by an edge move given by $H \cong G-u v+x w$ 
$u v \in E(G)$ and $\mathrm{x} w \in E(\bar{G})$. Later Johnson in [14] introduced a new kind of edge rotation called the "edge shift". It is defined as an edge rotation for the given graph $A, t=(u, v, w)$ such that $v w$ is an edge of A. As with an edge rotation, $t A$ will denote the newly formed graph $A-u v+u w$. This new edge rotation is again a metric.

Later an inequality between edge shift, edge rotation and edge move was proposed by Benade et al. [15]. The upper and the lower bounds were given by Faudree et al. in [16]. Under the concept of labeling, the distances between graphs using edge operation was done by Goddard et al. in [17].

The rotation distance between graphs $G$ and $H$ is denoted by $d_{r}(G, H)$, if there exists a sequence of graphs $G_{l}, G_{2}, \ldots, G_{k-l}$ such that $G_{l}$ is obtained by an edge rotation on $G$, and for each $1 \leq i \leq k-1, G_{i+l}$ is obtained by an edge rotation on $G_{i}$, with $H$ obtained from $G_{k-1}$ by one edge rotation. In this case we denote the rotation distance from $G$ to $H$ as $d_{r}(G, H)$ and it is equal to $k$.

Definition 1: [1] Let $S=G_{l}, G_{2}, \ldots, G_{k}$ be a set of graphs all of same order and the same size. Then the rotation distance graph $D(S)$ of $S$ has $S$ as its vertex set and vertices (graphs) $G_{i}$ and $G_{j}$ are adjacent if $d_{r}\left(G_{i}, G_{j}\right)$ $=1$, where $d_{r}\left(G_{i}, G_{j}\right)$ is the rotation distance between $G_{i}$ and $G_{j}$.

A graph $G$ is a edge rotation distance graph $(E R D G)$ (or r-distance graph) if $G \cong D(S)$ for some set of graphs.

A study done by Chartrand et al. [18] showed that the cycles, the complete bipartite graph $K_{3,3}$ and $K_{2, p}$ $(p \geq 1)$ are edge rotation distance graphs $(E R D G)$. This was later extended by Jarrett [19] in showing that complete graphs, trees and the wheel $\left(W_{l, n}\right)$ are also edge rotation distance graphs. Jarrett also gave a different proof other than the one due to Chartrand et al. [18]. It was shown that the complete bipartite graph $K_{m, n}(3 \leq m$ $\leq n)$ is a edge rotation distance graph.

In this paper we consider the edge rotations for the generalized Petersen graphs $\left(G_{p}(n, k)\right)$ and show that it is an edge rotation distance graph for $n \geq 5, n \in Z^{+}, k=1$ where $Z^{+}$is the set of all positive integers. We have also developed two algorithms which show the construction of distance degree injective graphs and almost distance degree injective graphs from cycles using the concept of edge rotation, some results on average eccentricity with respect to edge rotation are also proved.

\section{Edge Rotations for Generalized Petersen Graphs $G_{p}(n, k)$}

Watkins in [21].

The generalized Petersen graphs were introduced by Coxeter in [20] and later named by

Definition 2:For integers $n$ and $k$ with $2 \leq 2 \mathrm{k} \leq \mathrm{n}$, the generalized Petersen graph $G(n, k)$ has the vertex set $V(G(n, k))=u_{0}, u_{1}, \ldots, u_{n-l}, v_{0}, v_{l}, \ldots, v_{n-l}$ and the edge set $E(G(n, k))=\left[u_{i}, u_{i+j}\right],\left[u_{i}, v_{i}\right],\left[v_{i}, v_{i+k}\right]$, where $i$ is an integer and all subscripts are read modulo $n$.

As the name suggests $G(n, k)$ is generalization for the Petersen graph. In particular for $n=5, k=2$, we get the Petersen graph. Note that a generalized Petersen graph is a cubic graph.

$Z^{+}, k=1$.

In this section we consider edge rotations on the generalized Petersen graph where $n \geq 5, n \in$

The new graph obtained after a single edge rotation will be called as $G^{\prime}$, i.e., $G^{\prime}=G-e(G)+e(\bar{G})$

Theorem 2.1: An edge rotation in $G_{p}(n, 1)$ when $\mathrm{n}$ is odd results in a self-centered graph if the rotation of any edge induces a cycle of length 5 or 3 .

Proof: By the definition of rotation: $G^{\prime}=G-e(G)+e(\bar{G})$ where $G^{\prime}$ is the new graph obtained after a single edge rotation. As $G_{p}(n, l)$ is cubic graph, on rotation, a change in degrees of two vertices resulting in a nonregular graph. The girth of any generalized Petersen graph is always four. Hence upon rotation, $G^{\prime}$ induces a cycle of minimum length three to a maximum length (n-1). Among these, similar to the rotations shown in "Fig. 1 ", result in a self-centered graph. 

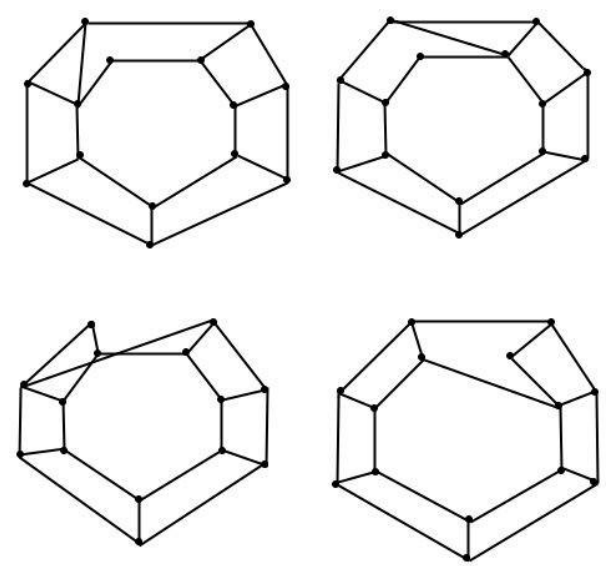

Fig 1: Rotations on a $G_{p}(7,1)$ graph

Remark 1: An edge rotation made on a $G_{p}(n, 1)$ when $\mathrm{n}$ is even does not allow the graph to be self-centered.

Remark 2: An edge rotation on $G_{p}(3,1)$ retains the radius thus resulting in a self-centered graph.

Remark 3: The complement of a circulant graph is not a Edge Rotation Distance Graph if the circulant consists of more than two jump sizes.

It was shown by E. B. Jarrett [19] that the cycles (for $n \geq 3$ ), the complete bipartite graph $K_{m, n}(3 \leq m \leq n)$ and the wheel $\left(W_{l, n}\right)$ is a $E R D G$. Now by a slight modification of the graphs used in proofs of the theorems proved by Jarrett in [19], we show that $G_{p}(n, k)$ where $k=1$ result in ERDG's. We first generate two cycles as in [19] and then show that the rotation distance graph on this class of cycles results in a generalized Petersen graph.

Theorem 2.2: The generalized Petersen graph $G_{p}(n, 1)$ where $n \geq 3$ and $n \in N$ is a edge rotation distance graph(ERDG).

Proof: Let $P$ be a path of length $3 n-1$ where $n$ is a natural number. Let the vertices of the path be denoted by $P: v_{0}, v_{1}, v_{2}, \ldots, v_{2}, v_{2 n+1}, \ldots, v_{3 n-l}$. Let $G$ be a graph obtained from $P$ by adding two new vertices $a_{l}, a_{2}$ and three new edges $v_{2 n+1} a_{1}, a_{1} a_{2}, a_{2} v_{2 n+1}$. Then for $i=1,2, \ldots, n-1$, we define $G_{i}$ to be the graph obtained from $G$ by adding two new vertices $x$ and $y$ such that $x$ is adjacent to $v_{2 i}$ and $y$ is adjacent to $v_{2 i+2}$. We also define $G_{n}$ as the graph obtained from $G$ by adding two new vertices namely $x$ adjacent only to $v_{2 n}$ and vertex $y$ adjacent only to $v_{2}$. 


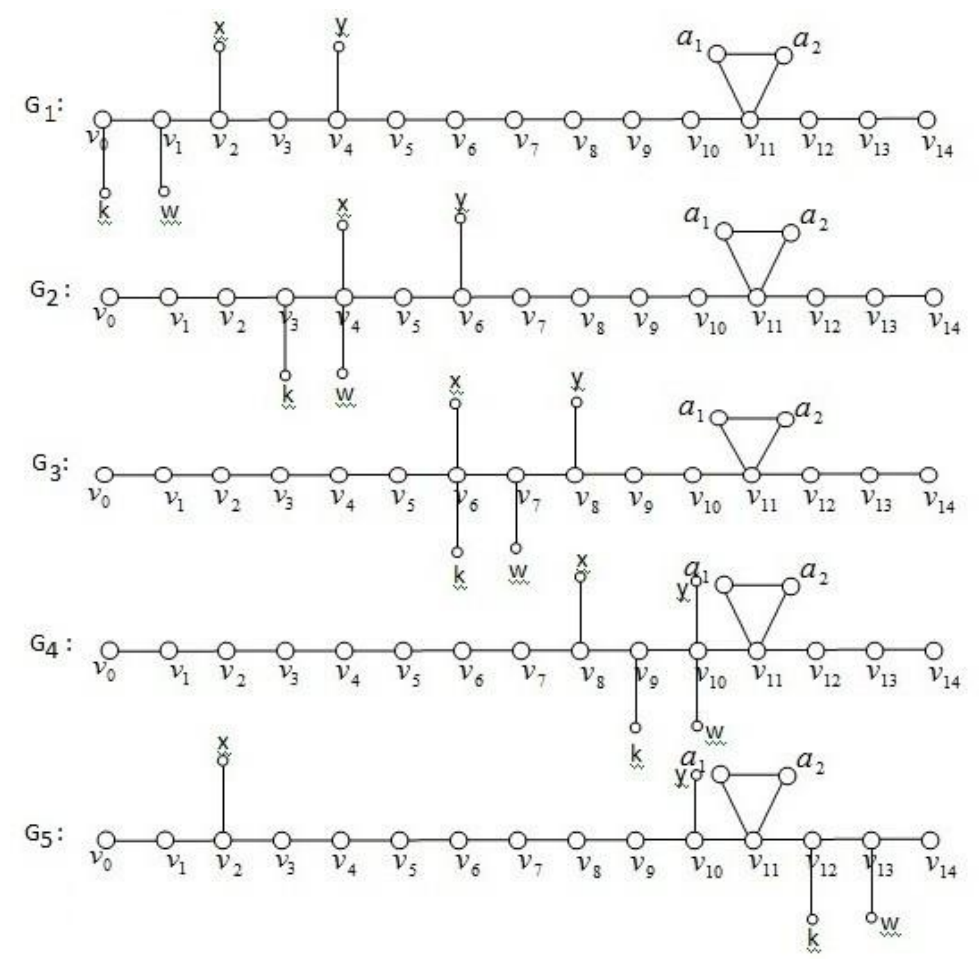

Fig 2: Rotation on graph G

Since an edge rotation changes the degrees of exactly two vertices $d_{r}\left(G_{i}, G_{j}\right)>1$ for integers $i$ and $j$, such that $1 \leq i \leq j \leq n$. On the other hand, for $i=1,2, \ldots, n-2$, the graph $G_{i+1} \cong G_{i}-x v_{2 i}+x v_{2 i+4}$ and consequently $\quad d_{r}\left(G_{i}, G_{i+1}\right)=1$. Similarly $\quad d_{r}\left(G_{n-1}, G_{n}\right)=d_{r}\left(G_{n}, G_{1}\right)=1$, since $G_{n} \cong G_{n-1}-x v_{2 n-2}+x v_{2}$ and $G_{1} \cong G_{n-1}-x v_{2 n}+x v_{4}$; thus $d_{r}\left(G_{l}, G_{2}, G_{3}, \ldots, G_{n}\right) \cong C_{n}$.

In a similar way we shall construct one more graph $H$ and consider the path to consists of vertices $P_{1}$ : $v_{0}, v_{1}, v_{2}, \ldots, v_{2}, v_{2 n+1}, \ldots, v_{3 n-1}$. Let $G$ be a graph obtained from $P_{1}$ by adding two new vertices $a_{1}, a_{2}$ and three new edges $v_{2 n+1} a_{1}, a_{1} a_{2}, a_{2} v_{2 n+1}$. Then for $i=1,2, \ldots, n-1$, we define $H_{i}$ to be the graph obtained from $H$ by adding two new vertices $x$ and $y$ such that $x$ is adjacent to $v_{2 i}$ and $y$ is adjacent to $v_{2 i+2}$. We also define $H_{n}$ as the graph obtained from $H$ by adding two new vertices namely $x$ adjacent only to $v_{2 n}$ and vertex $y$ adjacent only to $v_{2}$.

We now show that the edge rotation distance between each of these $G_{i}$ and $H_{i}$ is equal to one. To show that this relation exists we add two new vertices namely $w$ and $k$ and then rotate these edges such that $d_{r}\left(G_{l}, H_{i}\right)$ $=1$, for $i=1,2,3, \ldots, n$. 


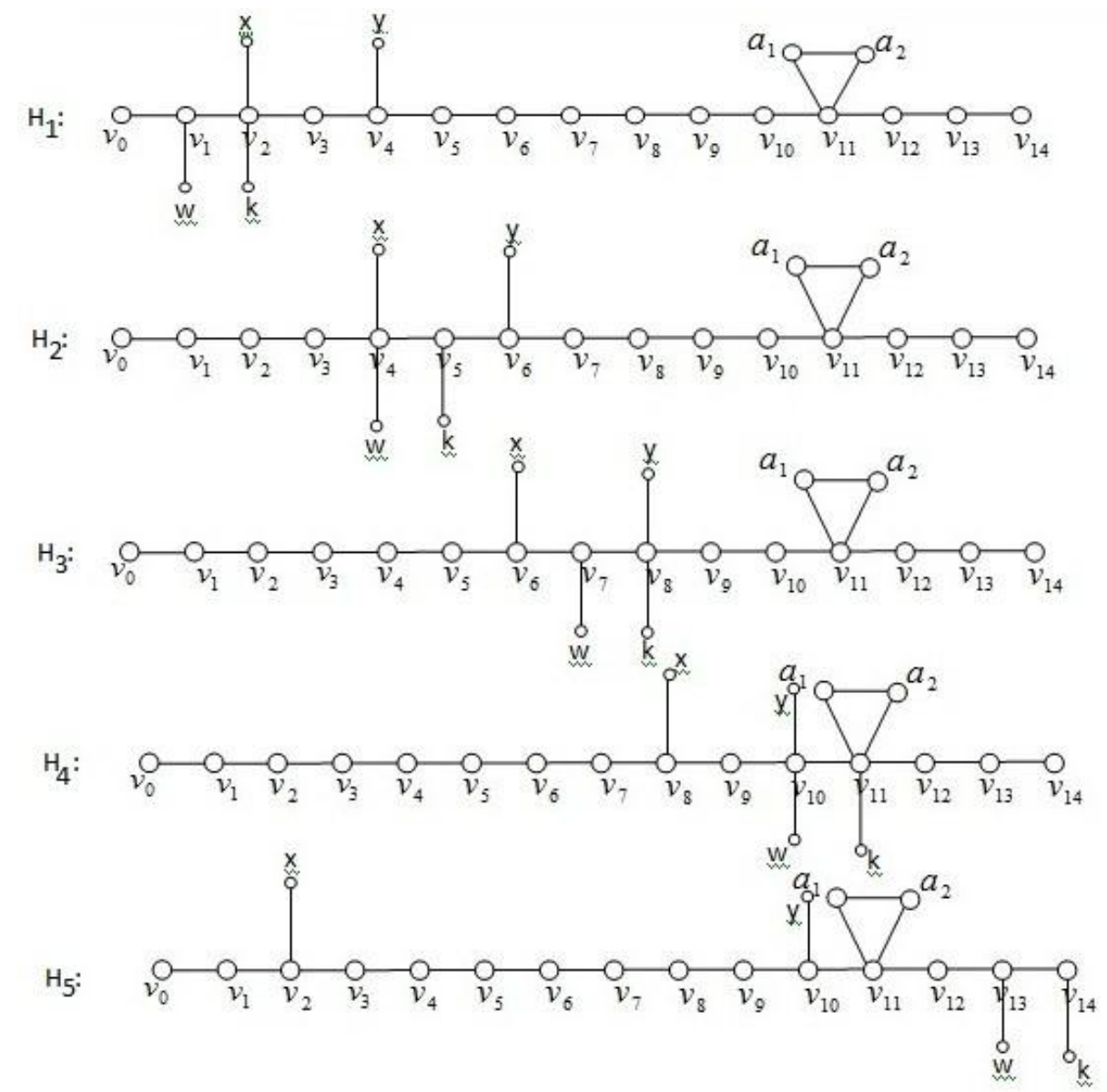

Fig 3: Rotations on graph $\mathbf{H}$

For $i=1,2, \ldots, n$, we first define $G_{1}$ to be the graph obtained from $G$ by adding two other vertices ' $k$ ' adjacent to $v_{0}$ and ' $w$ ' adjacent to $v_{l}$. The next graph $G_{2}$ is obtained by adding the vertices $k$ and $w$ adjacent to $v_{3}$ and $v_{4}$. In $G_{3}, k$ is adjacent to $v_{6}$ and $w$ is adjacent to $v_{7}$. The other $G_{i}$ 's are obtained in a similar pattern up to $i=$ $n-1$. In the last graph when $i=n$, the vertices $k$ and $w$ are adjacent to $3 n-3$ and $3 n-2$.

For $i=1,2, \ldots, n$, we first define $H_{l}$ to be the graph obtained from $H$ by adding two other vertices ' $w$ ' adjacent to $v_{l}$ and ' $k$ ' adjacent to $v_{2}$. The next graph $H_{2}$ is obtained by adding the vertices $w$ and $k$ adjacent to $v_{4}$ and $v_{5} . H_{3}$ is obtained by making $w$ adjacent to $v_{7}$ and $k$ adjacent to $v_{8}$. The other $H_{i}$ ' $s$ are obtained in a similar pattern up to $i=n-1$. In the last graph when $i=n$, the vertices $w$ and $k$ are adjacent to $3 n-3$ and $3 n-2$.

Since an edge rotation changes degrees of exactly two vertices $d_{r}\left(G_{l}, G_{i}\right)>1$. On the other hand for $i=$ $1,2,3, \ldots, n, H_{1} \cong G_{1}-k v_{0}+k v_{2}, \mathrm{H}_{2} \cong G_{2}-k v_{3}+k v_{5}$ and so on. Consequently, $d_{r}\left(G_{i}, H_{i}\right)=1$.

Hence, the rotation distance between each of $G_{i}$ and $H_{i}$ is always one.

We consider an example to generate a generalized Petersen graph for $\mathrm{n}=5$ and give an algorithmic construction of the graph which can be applied to higher order generalized Petersen graph.

Example 2.3Consider the graph $G_{p}(5,1)$

STEP - 1: For the graphs $G_{l}$ and $H_{1}$ obtained after adding the vertices $a_{1}, a_{2}$ which are used in the generation of the cycle, we add two new vertices namely ' $k$ ' and ' $w$ ' to $G_{l}$ and $H_{l}$. The adjacencies between these vertices is establishes as follows.

Join ' $k$ ' to $v_{0}$ and ' $w$ ' to $v_{l}$ in $G_{l}$. Now in $H_{l}$, join ' $w$ ' to $v_{l}$ and ' $k$ ' to $v_{2}$. In $G_{2}$, join ' $k$ ' to $v_{3}$ and ' $w$ ' to $v_{4}$. In $\mathrm{H}_{2}$, join ' $w$ ' to $v_{4}$ and ' $k$ ' to $v_{5}$. In $G_{3}$, join ' $k$ ' to $v_{6}$ and ' $w$ ' to $v_{7}$. In $\mathrm{H}_{3}$, join ' $w$ ' to $v_{7}$ and ' $k$ ' to $v_{8}$. In $G_{4}$, join ' $k$ ' to $v_{9}$ and ' $w$ ' to $v_{10}$. In $\mathrm{H}_{4}$, join ' $w$ ' to $v_{10}$ and ' $k$ ' to $v_{11}$. In $G_{5}$, join ' $k$ ' to $v_{12}$ and ' $w$ ' to $v_{13}$. In $\mathrm{H}_{4}$, join ' $w$ ' to $v_{13}$ and ' $k$ ' to $v_{14}$.

STEP - 2: Apply the concept of edge rotation to the newly added vertices. 
STEP - 3: We now rotate the newly added edges between each of $G_{i}$ and $H_{i}$. That is the edge $v_{0} k$ in $G_{l}$ is rotated to $v_{2} k$ in $H_{l}$ and thus $d_{r}\left(G_{l}, H_{l}\right)=1$.

In a similar way the rotation is carried in the rest of the graphs to establish the r-distance relation.

Also, $d_{r}\left(G_{2}, H_{2}\right)=d_{r}\left(G_{3}, H_{3}\right)=d_{r}\left(G_{4}, H_{4}\right)=d_{r}\left(G_{5}, H_{5}\right)=1=d_{r}\left(G_{1}, H_{1}\right)$.

STEP - 4: Thus, a relation is brought between the vertices of $G$ and $H$ in showing that the generalized Petersen graph is a $E R D G$.

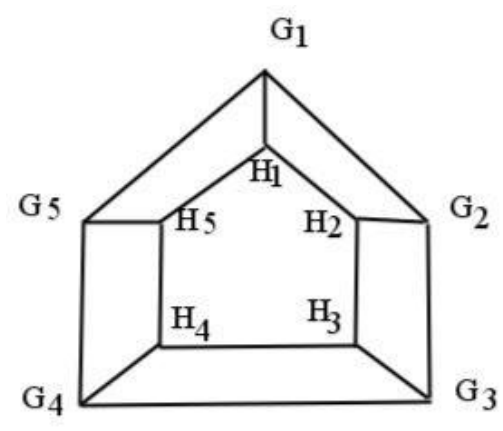

Fig 4: Edge rotation on $G_{p}(5,2)$

\section{Edge Rotation on $\mathrm{K}_{1, \mathrm{~m}}$}

Here we prove that the start $K_{l, m}$ is a edge rotation distance graph. The proof is an algorithmic one.

Lemma 3.1: The $\operatorname{star} K_{1, m}$, where $m \in Z^{+}$is a ERDG (for $m \geq 2$ ).

Proof: Consider the path $P$ of order $1+m$, where $1+m$ is the order of the star considered. We give a step by step procedure as proof to show that the star is a $E R D G$.

STEP - 1: Let $P=v_{1}, v_{2}, \ldots, v_{m+l}$ be a path and $G$ be the graph formed by adding two new vertices $v_{m+2}, v_{m+3}$.

STEP - 2: Join the vertices $v_{m+1} v_{m+2}, v_{m+2} v_{m+3}, v_{m+3} v_{m+1}$.

STEP - 3: Add a new vertex " $x$ " to $G_{i}, i=1,2, \ldots, m+1$ such that $x$ is adjacent to $v_{i}$ in $G_{i}$.

STEP - 4: $d_{r}\left(G_{l}, G_{l+k}\right)=1 . k=1,2, \ldots, m$.

That is $d_{r}\left(G_{l}, G_{2}\right)=d_{r}\left(G_{l}, G_{3}\right)=\ldots=d_{r}\left(G_{l}, G_{m}\right)=1$.

Here we observe that $\operatorname{deg}\left(v_{l}\right)$ in $G_{l}=2$. Similarly $\operatorname{deg}\left(v_{2}\right)$ in $G_{2}=3, \operatorname{deg}\left(v_{3}\right)$ in $G_{3}=3$. The presence of a degree two vertex in $G_{l}$ at $v_{l}$ changes the degree of that vertex upon rotation. There exists at least one vertex of degree three in each of $G_{2}$ to $G_{m+1}$ which is the result of edge rotation.

STEP - 5 : Thus the star $K_{l, m}$ is a ERDG.

Example 3.1:The following example shows that $K_{1,4}$ is a ERDG. Similar construction can be given to any positive integer $m$. 


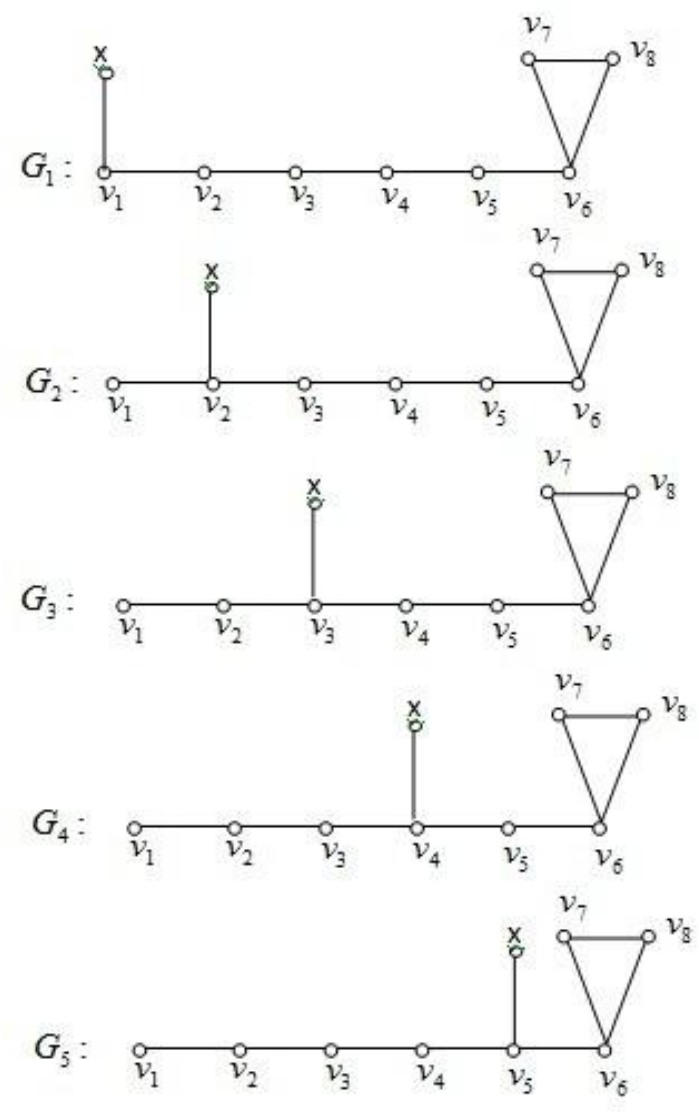

Fig 5:The star $K_{1, \mathrm{~m}}$ is a ERDG.

Remark 4:Let $G$ be a graph with a single cut - edge, then the graph becomes disconnected if the cut- edge is rotated to any other vertex on the same component.

Definition 3: [23] The eccentric mean or average eccentricity is defined as $\mu_{e}(G)=\left(\frac{1}{n}\right) \sum_{i=1}^{n} m_{i} e_{i}$ taken over all vertices in the graph where $m_{i}{ }^{\prime} s$ are the multiplicities of the eccentricities $e_{i}$.

Theorem 3.2 : For any even cycle $C_{n}$, for $n>4$, then average eccentricity of $C^{\prime}{ }_{n}$ lies between $\left[\frac{n}{2}-1, \frac{n}{2}+1\right]$ for any edge rotation in the cycle.

Proof: Let $u_{1}, u_{2}, \ldots, u_{n}, u_{1}$ be an even cycle. Let $u_{1} u_{2}$ be the edge to be rotated to any other vertex say $u_{3}, u_{4}, \ldots$ $\ldots, u_{n-1}$ or $u_{2} u_{1}$ be the edge to be rotated to any other vertex say $u_{4}, u_{5}, \ldots, u_{n}$. The rotation made induces a cycle of minimum length three to a maximum length $n$ - 1 . As their length of the cycle induced increases, the eccentricity of the vertices also increases. We observe that there exist at least two or more vertices which have the same eccentricity after the edge rotation. This occurs at the vertex where the rotation has been performed since this is the only vertex where we find two or more vertices with the same eccentricity and the rest of the vertices with different eccentricities. Thus we find that the average eccentricity of the graph $C_{n}{ }_{n}$ reduces by one upon rotation. Hence the eccentricity ranges from a minimum of $\frac{n}{2}-1$ to a maximum of $\frac{n}{2}+1$ upon different rotations. Thus when the average is taken over all the vertices we find that the average eccentricity lies between the closed interval $\left[\frac{n}{2}-1, \frac{n}{2}+1\right]$. 
Lemma 3.2 For any odd cycle $C_{n}$ the average eccentricity of $C_{n}^{\prime}{ }_{n}$ is not greater than $\left\lfloor\left(\frac{n}{2}\right)\right\rfloor+2$.

Proof : A single edge rotation induces a cycle of minimum length three and a maximum length $n-1$. The graph $C$ ' ${ }_{n}$ formed is a cycle followed by a path of some length " $m$ ". The eccentricity of each vertex ranges from $(n / 2)$ to $(n-2)$. Hence when the average is taken for the vertices of $C^{\prime}{ }_{n}$ we find that the value does not go beyond $\left\lfloor\left(\frac{n}{2}\right)\right\rfloor+2$.

Remark 5: Let $u_{1}, u_{2}, \ldots, u_{n}$ be a path. The average eccentricity becomes infinite and the graph becomes disconnected for the below mentioned rotations.

$$
\text { Avg Eccentricity }=\left\{\begin{array}{l}
u_{n-1} u_{n} \text { is rotated to } u_{1} \\
u_{1} u_{2} \text { is rotated to } u_{n}
\end{array}=\infty\right.
$$

Remark 6:A complete binary tree of " $n$ " levels requires at least one rotation to get transformed to a unicyclic graph.

Remark 7: Any tree on $\mathrm{n}$ vertices with $n \geq 5$ requires at least two rotations to become a unicyclic graph.

Lemma 3.3: The maximum number of rotations required for $K_{l, n}$ to be rotated to a path is $n-2$ for any natural number $\mathrm{n}$.

Proof: Leaving the last two edges or a $P_{3}$ the rest of the edges need to be rotated to form a path. Hence the number of edges that remain is $\mathrm{n}-2$. Thus $\mathrm{n}-2$ rotations are sufficient to transform to a path of length $\mathrm{n}$.

\section{Edge Rotations on Minimum Sized Self- Centered Graphs}

In this section we consider the minimal self-centered graphs of radius two proposed by Akiyama et al. [29]. We have checked these graphs for their radius and self-centeredness invariance after a single edge rotation. We also check on the average eccentricity of these graphs and give the bounds. Akiyama et al .[29] have constructed minimal self-centered graphs of radius two and shown that they one of the three classes given below.

(i) The Petersen graph $(5,2)$.

(ii) The graph obtained from the double star $S_{m, n}$ by adding a new vertex $v$ and joining $v$ to every end vertex of $S_{m, n}$.

(iii) The graph obtained from any $K_{3}(a, b, c)$ by joining a new vertex $w$ to each vertex of this $K_{3}(a, b, c)$.
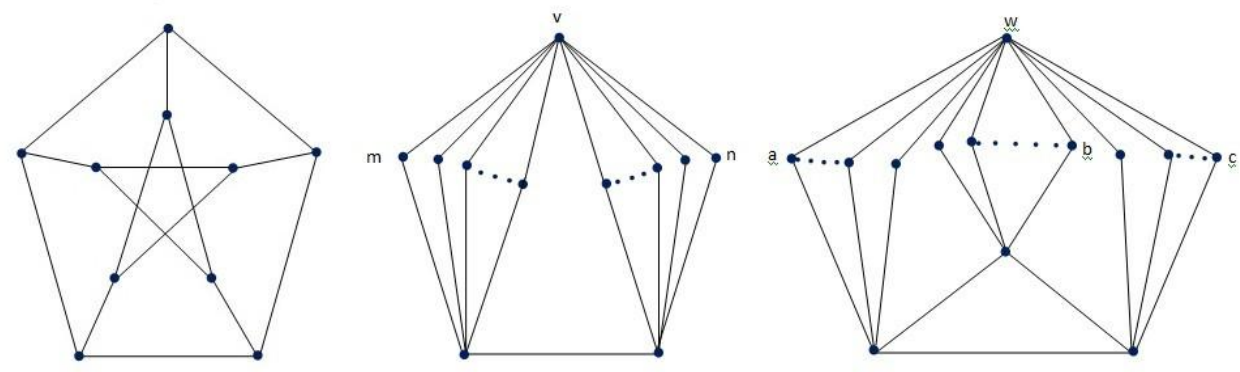

Fig 6: Minimal Self-centered graphs of radius two.

Lemma 4.1: If ' $m$ ' is the average eccentricity of any of the minimal self-centered graphs of radius two then the average eccentricity of $G$ ' is not more than $m+1$.

Proof: For the above mentioned three graphs we find that any rotation made increases the eccentricity of the vertices. Here we observe that when the edge operation is performed on Petersen graph, the eccentricity of at most two vertices increases by one since these exist other vertices whose eccentricity may increase or remain the same., Thus resulting the change in average eccentricity by at most one. Similar argument shows that the 
average eccentricity of the other two graphs increases by at most one. Thus the average eccentricity of the resulting graph does not exceed the value $m+1$.

\section{Algorithms}

In the next part we develop algorithms to generate DDI graphs/ ADDI graphs from an odd and even cycle by rotating a single edge. In literature the construction of DDI graphs is considered by Itagi Huilgol et al. [21]. But constructing a DDI graph from a minimum sized DDR graph viz., cycles is an interesting problem. Hence we give algorithms to construct DDI/ADDI graphs from cycles.

\section{Algorithm 5.1}

In the following algorithm we generate DDI/ADDI graphs from an odd cycle by rotating a single edge.

Let $C_{n}$ be a cycle of length $\mathrm{n}$.

STEP - 1: Input the cycle length $\mathrm{n}$ where $n \geq 5$.

STEP - 2: Find the eccentricity of the given cycle by using ecc $=\left\lfloor\left(\frac{n}{2}\right)\right\rfloor$.

STEP -3 : Consider the edge $e=u v$ to be rotated.

STEP - 4: Using the definition of rotation $G^{\prime}=G-e(G)+e(\bar{G})$ perform the edge rotation.

STEP - 5: If the newly added edge results in a cycle of length less than (or equal to) the eccentricity then the graph formed is a DDI graph.

else

If length $=$ ecc or ecc +1 , then it is a ADDI graph .

STEP $-6:$ If length $\geq e c c+2$, then it is neither a ADDI/DDI graph.

STEP -7 : Repeat steps 4 to 5 until length $>e c c+2$.

STEP $-8:$ STOP.

\section{Algorithm 5.2.} single edge.

In the second algorithm we develop ADDI graphs from evencycles using the concept of rotation of a

Let $C_{n}$ be a cycle of length $n$.

STEP - $1:$ Input the cycle length $\mathrm{n}$ where $n \geq 6$.

STEP -2 : Find the eccentricity of the given cycle by using $e=n / 2$.

STEP -3 : Consider the edge $e=u v$ to be rotated.

STEP -4 : Using the definition of rotation $G^{\prime}=G-e(G)+e(\bar{G})$ perform the edge rotation.

STEP $-5:$ If the newly added edge results in a cycle of length less thanthe eccentricity then the graph formed is a ADDI graph.

else

it is not a ADDI graph.

STEP -6 : Repeat steps 4 to 5 until length of the induced cycle is less than eccentricity.

STEP $-8:$ STOP. 


\section{Journal Papers:}

\section{References}

[1] G. Chartrand, F. Saba and H. B. Zou, Edge rotations and distances in graphs, Casopis pro pestovani matematiky, 110(1), 1985, 8791.

[2] Medha Itagi Huilgol, H. B. Walikar and B. D. Acharya, On diameter three Distance Degree Regular Graphs, Advances and Application in Discrete Math,7(1), 2011,39-61.

[3] Medha Itagi Huilgol, M. Rajeshwari and S. Syed Asif Ulla, Distance Degree Regular graphs and their eccentric digraphs, International Journal of Math. Sci and Eng. Appln., 5(VI),2011, 405-416.

[4] Medha Itagi Huilgol, M. Rajeshwari and S. Syed Asif Ulla, Products of DDR and DDI graphs, Journal of Discrete Mathematical Sciences and Cryptography,15(4:5),2012, 303-314.

[5] G. Brinkmann, Fast Generation of cubic graphs ,Journal of Graph Theory, 23(2),1996, 139-149.

[6] Medha Itagi Huilgol, M. Rajeshwari and S. Syed Asif Ulla, Embedding in distance degree regular and distance degree injective graphs, Malaya Journal of Matematik, 4(1),2013, 134-141.

[7] B. Zelinka, On a certain distance between isomorphism classes of graphs, Casopis pro pestovani matematiky, 100(4),1975, 371-373.

[8] B. Zelinka, A distance between isomorphism classes of graphs, Casopis propestovani matematiky, 33(1), 1983, 126-130.

[9] B. Zelinka, Comparision of various distances between isomorphism classes of graphs, Casopis pro pestovani matematiky, 110(3), 1985, 289-293.

[10] V. Balaz, J Koca, V. Kvasnika and M. Sekanina, A metric for graphs, Discrete Mathematics, 111(4), 1986, $431-433$.

[11] B. Zelinka, Edge distance between isomorphism classes of graphs, Casopis pro pestovani matematiky, 112(3),1987, $233-237$.

[12] B. Zelinka, The distance between a graph and its compliment, Casopis pro pestovani matematiky, 37(1),1987, 120-123.

[13] B. Zelinka, Contraction distance between isomorphism classes of graphs, Casopis pro pestovani matematiky, 115(2),1990, 211-216.

[14] M. Johnson, An ordering of some metrics defined on the space of graphs, Casopispro pestovani matematiky, 37(1),1987, 75-85.

[15] G. Benade, W. Goddard, T. A. Mckee and P. A. Winter, On distances between isomorphism classes of graphs, Mathematica Bohemica, 116(2), 1991, 160-169.

[16] R. J. Faudree, R. H. Schelp, L. Lesniak, A. Gyarfas and J. Lahel, On the rotation distance of graphs, Discrete Mathematics, North Holland, 126, 1994, 121-135.

[17] W. Goddard and H. C. Swart, Distance between graphs under edge operations,Discrete Mathematics, 161, 1996, $121-132$.

[18] G. Chartrand, W. Goddard, M. A. Henning, L. Lesniak, H. Swart and C. E. Wall, Which graphs are distance graphs?, Ars Combinatoria, 29A, 1990, 225-232.

[19] E. B. Jarrett, Edge rotation and edge slide distance graph, Computers Math. Applic., 34(11),1997, 81-87.

[20] H. S. M. Coxeter, Self-dual configurations and regular graphs, Bull. Ameri. Math. Soc., 56, 1950, 413-455.

[21] M. E. Watkins, A theorem on Tait Colorings with an applications to the generalized Petersen graphs, J. Combin. Theory, 6, 1969, $152-164$.

[22] Medha Itagi Huilgol and M. Rajeshwari, Non existence of cubic DDI graphs of diameter 4, 5, 6, (Communicated).

\section{Books:}

[23] F. Buckley and F Harary, Distance in Graphs (Addison Wesley, 1990)

[24] Gary Chartrand and Ping Zhang, Introduction to graph theory (Tata McGraw Hill, 2006)

\section{Proceeding Papers:}

[25] G. S. Bloom, L. V. Quintas and J. W. Kennedy, Some problems concerning distance and path degree sequence, Lecture Notes in Math, 1018 Springer - Verlag, Berlin,1983, 179-190.

[26] G. S. Bloom, L. V. Quintas and J. W. Kennedy, Distance Degree Regular Graphs, The theory and Applications of graphs, Fourth International conference, Western Michigan University, Kalamazoo, MI May 1980, John Wiley and Sons, New York, $1981,95-108$.

[27] F. Y. Halberstam and L.V. Quintas, A note on distance of tables and path degree sequence, Conference on Combinatorics, University of Waterloo, Waterloo, Canada, Jine 14 - July 21, 1982.

[28] J. Wolf, A small distance degree injective cubic graphs, Notesfrom New York graph theory day XIII , New York Academy of Sciences, 1987, 31-32.

[29] J. Akiyama, K. Ando and D. Avis, Miscellaneous properties of equi - eccentric graphs, Convexity and Graph Theory, Proc. Conf. in Haifa, Israel, 1981, North Holland, Amsterdam, 1984, 13-23. 